\title{
¿Existe el síndrome de burnout en los profesionales de la salud de las unidades de hemodiálisis de la región de Murcia?
}

\author{
Isabel Fernández Guzmán ${ }^{1}-M^{a}$ Carmen Fernández Márquez² - Rafael Rabadán Anta ${ }^{3}$ - Conrado Navalón Vila ${ }^{3}$ - \\ $M^{a}$ Dolores Martínez Espejo ${ }^{4}$
}

IPsicóloga, (Asociación de Ayuda al Enfermo Renal -ADAER-)

${ }^{2}$ Trabajadora Social, (Asociación de Ayuda al Enfermo Renal -ADAER-)

${ }^{3}$ Profesor Facultad de Psicología, (Universidad de Murcia)

${ }^{4}$ Profesora Facultad de Enfermería, (Universidad de Murcia)

\section{Resumen}

El contacto directo y continuo con pacientes renales en tratamiento sustitutivo con hemodiálisis así como con situaciones dónde el dolor, sufrimiento y desesperanza están presentes puede convertirse en una fuente de estrés y conducir al desarrollo de lo que se ha denominado "síndrome de estar quemado" o Burnout.

El objetivo principal de este estudio fue medir el nivel de Burnout, la ansiedad y depresión en los profesionales de todas las unidades de diálisis de la Región de Murcia, así como su relación con la edad, tipo de unidad en la que se trabaja (pública o privada concertada) y tiempo en la profesión. Resulta necesaria la prevención y detección de este síndrome por las consecuencias que puede llegar a tener en el profesional.

El estudio realizado es de tipo descriptivo, transversal y multicéntrico. Los instrumentos utilizados fueron

\section{Correspondencia:}

Isabel Fernández Guzmán

Asociación Ayuda al Enfermo Renal (ADAER)

C/ De la Merced, 12. Entlo. A y B. 30.001 (Murcia)

E-mail: ifernandezguzman@yahoo.es el Cuestionario para la Evaluación del Síndrome de Quemarse por el Trabajo de Gil-Monte, el Inventario de Ansiedad Rasgo-Estado de Spielberger y el Inventario de Depresión de Beck.

El análisis de los resultados obtenidos pone de manifiesto que el nivel global de burnout entre los profesionales de la nefrología de la Región de Murcia se sitúa en un nivel medio-bajo ( $M d n=1.13)$, encontrándose diferencias marginalmente significativas ( $p=.082$ ) entre aquellos que trabajan en unidades públicas y los que desarrollan su profesión en clínicas concertadas, que presentaron un promedio ligeramente superior. Asimismo se constatan diferencias significativas $(p<.0001)$ entre los distintos centros de diálisis de la Región.

\section{PALABRAS CLAVE:}

- BURNOUT: SÍNDROME DE ESTAR QUEMADO

- PERSONAL SANITARIO

- HEMODIÁLISIS

- CESQT-PS

- ANSIEDAD

- DEPRESIÓN 


\section{Does burnout syndrome exist in healthcare professionals in the haemodialysis units in the Murcia region?}

\begin{abstract}
Direct, continuous contact with renal patients undergoing replacement therapy involving haemodialysis and with situations where pain, suffering and despair are present can become a source of stress and lead to the development of what has been called "burnout syndrome".

The main object of this study was to measure the level of burnout, anxiety and depression in the professionals in all the dialysis units in the Murcia Region, and the relationship with age, type of unit where the professional works (public or publiclycontracted private) and time in the profession. The prevention and detection of this syndrome is necessary on account of the consequences it can have for the professional.
\end{abstract}

The study carried out is descriptive, transversal and multicentric. The instruments used were the Gil-Monte Questionnaire for Evaluation of Burnout Syndrome, the Spielberger State-Trait Anxiety Inventory and the Beck Depression Inventory .

The analysis of the results obtained shows that the overall level of burnout among nephrology professionals in the Murcia Region is medium-low $(M d n=1.13)$, with marginally significant differences being found $(p=.082)$ between those who work in public units and those employed in publiclycontracted private clinics, who showed a slight higher mean. Significant differences $(p<.0001)$ were also observed between the different dialysis centres in the Region.

\section{KEY WORDS:}

- BURNOUT

- HEALTHCARE PERSONNEL

- HAEMODIALYSIS

- CESQT-PS

- ANXIETY

- DEPRESSION

\section{Introducción}

Ser profesional de la salud conlleva la exposición a situaciones complejas que pueden generar estados de tensión por infinidad de factores entre los que cabe destacar, que gran parte del trabajo se desarrolla en contacto directo con personas que tienen problemas de salud y con sus familiares, manteniendo una relación muy estrecha con el dolor, el sufrimiento y la desesperanza. Por esta razón, el ámbito laboral puede convertirse en una fuente constante de estrés y conducir al desarrollo de lo que se ha denominado "síndrome de estar quemado" (SQT) o Burnout ${ }^{1}$.

El concepto de burnout fue utilizado por primera vez en Estados Unidos a mediados de los años setenta por Freudenberguer ${ }^{2}$ para describir el desgaste que experimentaban determinados profesionales en situaciones de estrés laboral. Han sido muchos los autores que posteriormente han descrito y estudiado este síndrome pudiendo destacar la aportación de Maslach y Jackson ${ }^{3}$ que lo conceptualizaron como: "Una respuesta al estrés laboral crónico integrado por actitudes y sentimientos negativos hacia las personas con las que se trabaja y hacia el propio rol profesional, así como por la vivencia de encontrarse emocionalmente agotado". Esta respuesta se caracteriza por un deterioro cognitivo, consistente en la pérdida de la ilusión por el trabajo, el desencanto profesional, o la baja realización personal en el trabajo; por un deterioro afectivo caracterizado por agotamiento emocional y físico; y por la aparición de actitudes y conductas negativas hacia los pacientes. A veces, estos síntomas se acompañan de sentimientos de culpa, variable clave para entender la aparición de algunas de las consecuencias más importantes del burnout, como depresión o absentismo.

Existen diferentes modelos explicativos del SQT, pudiendo destacar en la actualidad los de tipo transaccional que plantean la experiencia de desgaste profesional como consecuencia de la interacción de determinadas variables del entorno laboral y las características de personalidad ${ }^{4,5}$.

Entre las principales consecuencias de este síndrome se encuentra la aparición de problemas tanto a nivel personal (físicos: e.g. insomnio, cefaleas y problemas musculares, entre otros/, y psicológicos: e.g. ansiedad y depresión), familiar (aislamiento e irritabilidad ante los demás) y laboral (absentismo, mínima implicación 
y despersonalización). Por esto podemos afirmar que dicho síndrome repercute directamente en el profesional e indirectamente sobre los usuarios o pacientes, ya que se puede ver afectada la calidad de los cuidados recibidos.

El burnout ha sido estudiado en muchas profesiones de ayuda (voluntariado, educadores, servicios sociales y sanitarios) y existen numerosos estudios en profesionales de enfermería ${ }^{6,7,8}$ siendo mucho más escasos en la especialidad de enfermería nefrológica9,10,11.

Los profesionales de la salud participantes en este estudio desarrollan su actividad atendiendo a los enfermos renales en tratamiento sustitutivo con hemodiálisis, compartiendo un número elevado de horas a la semana con dichos pacientes, ya que como mínimo son tres las sesiones semanales a las que deben someterse, con una duración de cuatro horas por sesión. Uno de los pilares básicos del mantenimiento de un equilibrio en el paciente crónico en general y del paciente renal en particular, va a ser la relación con los profesionales que le atienden.

Las unidades de hemodiálisis presentan características que las hace especialmente vulnerables a la aparición de estrés, siendo las tres características principales, según Climent y Mendes Diz ${ }^{12}$, la continuidad (los pacientes acuden con frecuencia semanal y a lo largo del tiempo), la contingencia (por las características de la enfermedad y tratamiento existe la necesidad de resolver problemas que surgen de forma imprevista) y la alta emotividad (el personal trabaja permanentemente frente al dolor, el sufrimiento y la muerte, lo cual crea un clima de gran estrés emocional). A todo lo citado hasta aquí hay que añadir que el enfermo renal, como paciente crónico, frecuentemente presenta una serie de alteraciones emocionales y de comportamiento que también puede repercutir en el personal que lo atiende.

\section{Objetivos}

Los principales objetivos que pretenden conseguirse a través de este estudio son:

1. Medir el grado de ansiedad, depresión y "síndrome de quemarse por el trabajo" en el personal de todas las unidades de diálisis de la Región de Murcia.
2. Relacionar el nivel de Burnout con la edad, tipo de unidad en la que se trabaja (pública o privada concertada), y tiempo en la profesión.

\section{Metodología}

El estudio que planteamos es de tipo descriptivo, transversal y multicéntrico, cuyo ámbito de aplicación son todos los profesionales de las unidades de diálisis de la Comunidad Autónoma de Murcia. En el momento de la recogida de datos existían doce unidades de hemodiálisis ubicadas en diferentes puntos de la Región.

\section{Procedimiento}

Para poder realizar este estudio, en primer lugar se mantuvo una reunión con cada uno de los supervisores de los centros de hemodiálisis de la Región de Murcia, en la que se explicó el propósito de la investigación. Obtenido el consentimiento, se proporcionó al supervisor de cada unidad de diálisis un sobre para cada uno de los profesionales sanitarios, el cual incluía una carta de presentación en la que se explicaba el objetivo del estudio y se pedía su colaboración especificando su carácter anónimo, y un ejemplar de cada uno de los cuestionarios utilizados (CESQT-PS, BDI y STAI). El plazo máximo propuesto para la recogida de los cuestionarios fue de un mes.

De los 310 sobres remitidos a todas las unidades de la región murciana, 197 fueron cumplimentados correctamente, obteniéndose así una aceptable tasa de respuesta total del $64 \%$. Atendiendo a su distribución por colectivos, el grupo de enfermería fue el que más colaboró (74\%), seguido por auxiliares de enfermería (58\%) y, finalmente, el colectivo de médicos (24\%).

\section{- Participantes}

La muestra estuvo formada por profesionales sanitarios de los diferentes centros de diálisis de toda la Región de Murcia, tanto públicos como concertados. Este colectivo lo integran médicos, enfermeros y auxiliares $(N=197)$.

En cuanto a las características descriptivas de la muestra, según el tipo de centro 38 profesionales trabajaban 
en hospitales públicos (19\%), mientras que 159 lo hacían en centros concertados (81\%). En función del sexo, 34 eran hombres (17\%) y 163 mujeres (83\%). Para la variable edad, 105 profesionales tenían entre 20 y 30 años, 45 entre 31 y 40 años, 41 entre 41 y 50 años, y 6 entre 51 y 65 años. En cuanto a la profesión, 9 eran médicos, 130 enfermeros y 58 auxiliares. De todos los profesionales que colaboraron, el 49\% llevaban trabajando en su respectiva profesión un máximo de 5 años, el $27 \%$ llevaban entre 5 y 10 años, el $13 \%$ entre 10 y 20 años y tan sólo un $11 \%$ más de 20 años.

\section{- Instrumentos}

\section{Cuestionario para la Evaluación del Síndrome de Quemarse por el Trabajo (CESQT-PS) de Gil-Mon- $t^{13}$}

Este instrumento consta de 20 ítems valorados con una escala tipo Likert (0-4) que se distribuyen en cuatro dimensiones, denominadas: Ilusión por el trabajo ( 5 ítems, $\alpha=.82$ ), Desgaste psíquico (4 ítems, $\alpha=.83$ ), Indolencia (6 ítems, $\alpha=.75$ ) y Culpa ( 5 ítems, $\alpha=.73$ ). EI CSQT ha obtenido buenos resultados de fiabilidad y validez en estudios previos ${ }^{14,15}$.

A efectos diagnósticos se considera que un individuo ha desarrollado el SQT cuando presenta altas puntuaciones en el CESQT, calculadas como promedio de los ítems Ilusión por el trabajo (los ítems que componen esta dimensión están formulados de manera positiva, por lo que bajas puntuaciones indican un alto nivel de burnout), Desgaste psíquico e Indolencia. No se incluyen los ítems de la escala de Culpa debido a que no todos los individuos desarrollan estos sentimientos aunque puntúen alto en el cuestionario. Se consideran casos graves de SQT, con probable deterioro de la salud, aquellos que presentan altas puntuaciones en el CESQT junto con altos sentimientos de Culpa ${ }^{16}$.

\section{Inventario de Depresión de Beck (BDI) ${ }^{17}$}

Se trata de una herramienta ampliamente utilizada para la evaluación de la depresión por los profesionales de la salud y consta de 21 ítems de opción múltiple que evalúan un amplio espectro de síntomas. El marco temporal hace referencia al momento actual y a la semana previa. En su versión actual, el cuestionario está diseñado para personas mayores de 13 años, y se compone de elementos correspondientes a los síntomas de la depresión como la desesperanza y la irritabilidad, cogniciones como la culpa o sentimientos de ser castigados, así como síntomas físicos del tipo fatiga, pérdida de peso y falta de interés en el sexo.

EI BDI establece diferentes puntos de corte para valorar la intensidad y severidad de la sintomatología presentada. Sus índices psicométricos han sido estudiados de manera casi exhaustiva, mostrando una buena consistencia interna $(\alpha=.76)$.

\section{Cuestionario de Ansiedad Estado-Rasgo (STAI) de Spielberger ${ }^{18}$.}

La prueba consta de dos partes, con 20 cuestiones cada una de ellas. La primera, ansiedad-estado (A/E), evalúa un estado emocional transitorio, caracterizado por sentimientos subjetivos, conscientemente percibidos, de atención y aprensión y por hiperactividad del sistema nervioso autónomo. La segunda, ansiedad- rasgo $(A / R)$, señala una propensión ansiosa, relativamente estable, que caracteriza a los individuos con tendencia a percibir las situaciones como amenazadoras.

Los análisis factoriales han mostrado una buena consistencia interna ( $\alpha=.84)$.

La tabulación de datos y su análisis estadístico se ha realizado con el programa SPSS 15.0 para Windows.

Se aplicaron diversos análisis de varianza (ANOVA) y de correlación bivariada de Pearson con el objetivo de determinar si resulta significativo el nivel de burnout, así como de explorar las relaciones existentes entre distintas variables del estudio.

\section{Resultados}

El nivel global de burnout entre los profesionales de la nefrología de la Región de Murcia se sitúa en un nivel medio-bajo ( $M d n=1.13)$, encontrándose diferencias marginalmente significativas $(p=.082)$ entre aquellos que trabajan en unidades públicas y los que desarrollan su profesión en clínicas concertadas, que presentaron un promedio ligeramente superior. Asimismo se constatan diferencias significativas $(p<.0001)$ entre los distintos centros de diálisis de la Región (Tabla 1).

De las variables recogidas que pueden estar relacionadas con el nivel de burnout presentado, el sexo y el tiempo que los profesionales llevan desarrollando su 


\begin{tabular}{|l|c|c|c|}
\hline \multicolumn{1}{|c|}{ Centros diálisis } & Media & N & \\
\hline BURNOUT GLOBAL &, 9674 & 19 & Desviación típica \\
\hline Arrixaca & 1,4033 & 27 &, 42519 \\
\hline El Palmar & 1,1000 & 12 &, 69152 \\
\hline Rafael Méndez &, 9243 & 7 &, 53843 \\
\hline El Rosell &, 9314 & 7 &, 51221 \\
\hline Yecla & 1,4527 & 15 &, 64814 \\
\hline Caravaca & 1,3143 & 23 &, 66044 \\
\hline Nefroclub Carthago & 1,1838 & 8 &, 38840 \\
\hline San Pedro Pinatar & 1,1267 & 30 &, 40699 \\
\hline Ronda Sur &, 5350 & 14 &, 43163 \\
\hline Hemolorca & 1,1229 & 28 &, 56804 \\
\hline Molina Segura & 1,2214 & 7 &, 44890 \\
\hline Cieza & 1,1433 & 197 &, 56064 \\
\hline TOTAL & & & \\
\hline
\end{tabular}

Tabla 1. Nivel de burnout por centros

profesión no aparecen como estadísticamente significativas, mientras que las diferencias encontradas en la variable edad sí que lo son ( $p=.033)$, observándose un descenso gradual en las puntuaciones de burnout conforme se asciende en los intervalos de edad.

Un $11 \%$ de los profesionales que participaron en el estudio presentan un cuadro de depresión moderada que tras el análisis realizado cursa con un mayor nivel de burnout $(p=.000)$. Entre los grupos profesionales, los enfermeros son los que presentan un mayor nivel de depresión y burnout.

No todos los grupos profesionales presentan el mismo nivel de burnout, destacando significativamente $(p=.018)$ el colectivo de enfermería con un promedio de 1.21 , seguido por los auxiliares y presentando el nivel más bajo los médicos; las comparaciones posthoc por pares, presentan diferencias entre médicos y enfermeros y entre médicos y auxiliares (Tabla 2).

\begin{tabular}{|c|c|c|c|c|c|c|}
\hline \multicolumn{7}{|c|}{ COMPARACIONES MÚTIPLES } \\
\hline \multicolumn{7}{|c|}{$\begin{array}{l}\text { Variable dependiente: Burnout Global } \\
\text { DMS }\end{array}$} \\
\hline \multirow{2}{*}{ (I) Profesión } & \multirow{2}{*}{ (J) Profesión } & \multirow{2}{*}{ Diferencia de medias (I-J) } & \multirow{2}{*}{ Error típico } & \multirow{2}{*}{ Sig. } & \multicolumn{2}{|c|}{ Intervalo de confianza al (95\%) } \\
\hline & & & & & Límite inferior & Límite superior \\
\hline Médico & $\begin{array}{l}\text { Enfermero/a } \\
\text { Auxiliar }\end{array}$ & $\begin{array}{l}-, 51098^{*} \\
-, 39117\end{array}$ & $\begin{array}{l}19085 \\
, 19856\end{array}$ & $\begin{array}{l}, 008 \\
, 050\end{array}$ & $\begin{array}{l}-, 8874 \\
-, 7828\end{array}$ & $\begin{array}{l}-, 1345 \\
, 0005\end{array}$ \\
\hline Enfermoro/a & $\begin{array}{l}\text { Médico } \\
\text { Auxiliar }\end{array}$ & $\begin{array}{l}\text {,51098* } \\
, 11981\end{array}$ & $\begin{array}{l}19085 \\
, 08804\end{array}$ & $\begin{array}{l}, 008 \\
, 175\end{array}$ & $\begin{array}{r}, 1345 \\
-, 0538\end{array}$ & $\begin{array}{l}, 8874 \\
, 2935\end{array}$ \\
\hline Auxiliar & $\begin{array}{l}\text { Médico } \\
\text { Enfermero/a }\end{array}$ & $\begin{array}{r}39117 \\
-, 11981\end{array}$ & $\begin{array}{l}19856 \\
, 08804\end{array}$ & $\begin{array}{l}, 050 \\
, 175\end{array}$ & $\begin{array}{l}-, 0005 \\
-, 2935\end{array}$ & $\begin{array}{l}, 7828 \\
, 0538\end{array}$ \\
\hline
\end{tabular}

Tabla 2. Nivel de burnout por colectivo profesional 
En la medida de depresión no se constatan diferencias significativas $(p=.478)$ entre médicos, enfermeros y auxiliares. En el nivel de ansiedad-estado presentado sí que se existen diferencias marginalmente significativas $(p=0.92)$ entre los grupos profesionales, destacando en este caso el nivel presentado por los auxiliares. Le siguen los enfermeros y médicos.

Como era esperable, el burnout presenta correlaciones positivas significativas con depresión ( $R=.385 ; p=.000)$, ansiedad-estado ( $R=.412, p=.000)$ y ansiedad-rasgo $(\mathrm{R}=.372, \mathrm{p}=.000)$; véase (Tabla 3$)$.

A nivel descriptivo, podemos establecer el perfil del profesional que presenta un mayor nivel de burnout como el de enfermero/a entre 20 y 30 años empleado en clínica privada y que manifiesta depresión moderada.

\section{Discusión y conclusiones}

La tasa de respuesta en esta investigación engloba amplias variaciones entre hospitales públicos (38\%) y clínicas concertadas (62\%) con valores similares a los que se describen en distintos estudios realizados sobre el mismo tema ${ }^{11,19}$.

Los principales resultados obtenidos en las evaluaciones de la intervención realizada nos permite concluir que actualmente no existe un nivel significativo de burnout entre los profesionales de nefrología de la Región aunque consideramos que debe promoverse la aplicación de programas preventivos debido a que el contacto continuo con pacientes crónicos puede conllevar la aparición de estrés, así como a que el intervalo de edad más habitual de los profesionales (20-30 años) puede considerarse un periodo de sensibilización al SQT4

Resulta llamativa la existencia de diferencias entre las unidades de diálisis, lo que abre la posibilidad de analizar en un nuevo estudio qué factores pueden estar relacionados con este hecho, así como de obtener datos para valorar longitudinalmente la evolución de este síndrome.

Recibido: 30 Junio 2011

Revisado: 14 septiembre 2011

Modificado: 29 Diciembre 2011

Aceptado: 25 Enero 2012

\section{Bibliografía}

1. Rodríguez-Marín J. Psicología Social de la Salud. Madrid: Síntesis; 1995.

2. Freudenberger HJ. Staff Burnout. Journal of Social Issues. 1974; 30(1):159-165.

\begin{tabular}{|c|c|c|c|c|c|}
\hline & & Burnout Global & Depresión & A. Estado (Pc) & A. Rasgo (Pc) \\
\hline \multirow[t]{3}{*}{ Burnout Global } & Correlación de Pearson & 1 &, $385(* *)$ &, $412(* *)$ & $372(* *)$ \\
\hline & Sig. (bilateral) & &, 000 &, 000 &, 000 \\
\hline & $\mathrm{N}$ & 197 & 196 & 196 & 196 \\
\hline \multirow[t]{3}{*}{ Depresión } & Correlación de Pearson &, $385(* *)$ & 1 &, $631(* *)$ &, $712\left(^{* *}\right)$ \\
\hline & Sig. (bilateral) &, 000 & &, 000 &, 000 \\
\hline & $\mathrm{N}$ & 196 & 196 & 195 & 195 \\
\hline \multirow[t]{3}{*}{ A. Estado (Pc) } & Correlación de Pearson &, $412(* *)$ &, $631(* *)$ & 1 &, $803(* *)$ \\
\hline & Sig. (bilateral) &, 000 &, 000 & &, 000 \\
\hline & $\mathrm{N}$ & 196 & 195 & 196 & 196 \\
\hline \multirow[t]{3}{*}{ A. Rasgo (Pc) } & Correlación de Pearson &, $372(* *)$ &, $712(* *)$ & $803(* *)$ & 1 \\
\hline & Sig. (bilateral) &, 000 &, 000 & ,000 & \\
\hline & $\mathrm{N}$ & 196 & 195 & 196 & 196 \\
\hline
\end{tabular}

Tabla 3. Correlaciones entre burnout, depresión y ansiedad (estado/rasgo) 
3. Maslach C, Jackson SE. Maslach Burnout Inventory. Palo Alto, CA: Consulting Psychologists Press; 1981.

4. Gil-Monte PR, Peiró JM. Desgaste psíquico en el trabajo: El síndrome de quemarse. Madrid: Síntesis; 1997.

5. Moreno-Jiménez B, Rodríguez-Muñoz A, GarrosaHernández E, Morante-Benadero ME. Antecedentes organizacionales del acoso psicológico en el trabajo: un estudio exploratorio. Psicothema. 2005; 17(4): 648-653.

6. Mallet KL. The relationships between Burnout, death anxiety, and social suport in hospice and criticial care nurses. Ann. Arbor, Michigan: University Microfilms International (UMI). Bell \& Howell Information; 1991.

7. García M. Burnout en profesionales de enfermería de centros hospitalarios. Revista de Psicología del trabajo y las organizaciones.1991; 7(18): 3-12.

8. Schaufeli WB, Janczur B. Burnout among nurses: A Polish-Dutch comparison. Journal of Cross-Cultural Psychology.1994; 25(1): 95-113.

9. Lewis $S L$, Campbell MA. Work, stress and burnout, and sense of coherence among dialysis nurses. Anna Journal. 1992; 19(6): 545-553.

10. Fernández-Viña A, Reguera R, Amigo I, Vallejo G. Estudio sobre la afectación del Burnout en el personal de enfermería de los servicios de hemodiálisis, oncología y cirugía general. Libro de comunicaciones XXII Congreso Nacional SEDEN. 1997:250-252.

11. Del Campo Romero C, Fernández-Repeto Valls E, Martínez Delgado JM, Rojas Blanco A. El síndrome de quemarse por el trabajo (Burnout) en los profesionales de enfermería de las unidades de diálisis de la Provincia de Cádiz. Libro de comunicaciones al XXIV Congreso Nacional SEDEN. 1999: 194-199.

12. Climent $G$, Mendes Diz A. Modelo para la definición y evaluación de la calidad del personal de enfermería. Medicina y Sociedad. 1983: 6 (6).

13. Gil-Monte PR. Cuestionario para la evaluación del Síndrome de Quemarse por el Trabajo (CSQT). Madrid:TEA Ediciones; 2011.

14. Unda S, Sandoval JI, Gil-Monte PR. Prevalencia del síndrome de quemarse por el trabajo (SQT) (burnout) en maestros mexicanos. Informació Psicológica. 2008; 91 (92): 53-63.

15. Marucco MA, Gil-Monte PR, Flamenco E. Síndrome de quemarse por el trabajo (burnout) en pediatras de hospitales generales, estudio comparativo de la prevalencia medida con el MBI-HSS y el CESQT. Informació Psicológica. 2008; 91(92): $32-42$.

16. Gil-Monte PR, Carretero N, Roldán MD, NúñezRomán E. Prevalencia del síndrome de quemarse por el trabajo (burnout) en monitores de taller para personas con discapacidad. Revista de Psicología del Trabajo y de las Organizaciones. 2005; 21(1-2): 107-123.

17. Beck AT. Depression Inventory. Philadelphia: Center for Cognitive Therapy; 1978.

18. Spielberger $C D$, Gorsuch $R L$, Lushene R. Manual del Cuestionario de Ansiedad Estado/Rasgo, $3^{\mathrm{a}}$ ed. Madrid:TEA Ediciones; 1982.

19. Atance JC. Aspectos epidemiológicos del Síndrome de Burnout en personal sanitario. Rev. Esp. Salud Pública. 1997; 71(3): 293-303. 